\title{
The Relationship Between Intra/Extra-familial Childhood Sexual Abuse and Survivors' Mental Health
}

\author{
Magnolia Bahle Ngcobo-Sithole \\ Department of Psychology and Department of Psychiatry, Stikland Psychiatry Hospital and Stellenbosch University, South Africa
}

Copyright $@ 2015$ Horizon Research Publishing All rights reserved.

\begin{abstract}
The impact of intra-familial sexual abuse and extra-familial sexual abuse on children is the focus of this article. Intra-familial childhood sexual abuse is a common occurrence within the African population but its impact is not reported. A sample of sexually abused children referred for psychological intervention in a general hospital in South Africa was assessed for symptoms of depression, PTSD, and cognitive deficits. The results showed that while most of the children present with subclinical symptoms of depression and posttraumatic stress disorder they scored below average on most tests of cognitive functioning. Psychological theories are used to conceptualize the results.
\end{abstract}

Keywords Sexual Abuse, Intra-familial Sexual Abuse, Extra-familial Sexual Abuse, Childhood Sexual Abuse, Depression, Posttraumatic Stress Disorder, Cognitive Functioning

\section{Introduction}

Sexual abuse is one of the most common traumatic experiences in childhood. In predominantly black communities it has become more common than previously thought. The prevailing myth has been that children are raped or molested by a stranger. Recent studies show that sexual assault is now widely prevalent in domestic settings. Suggesting that the perpetrator can be a family member (e.g. father, step father, uncle, grandparent)

When children are sexually violated by someone close to them it can be very devastating for both the child and the entire family. Knowing that the perpetrator is loved by other members of the family complicates matters even more. The child sits with the problem and engages in self blaming behaviours rather than dare to disclose. Cultural beliefs among black communities pose more challenges. Virtually every culture has spoken and unspoken rules about sex and sexuality. These rules can make it even more difficult for children to ask for help when they have been sexually violated. For example, in cultures that place a high value on female virginity like the Zulu culture, a girl who has been sexually abused may feel that she has been disgraced and is now "damaged goods" who no one would want to marry. This can lead to feelings of shame that in turn lead to further secrecy and opens doors for mental health problems in the child. The literature provides contradictory results regarding the identity of the perpetrator. For example in an American study Kilprak and colleagues [1] found that strangers were responsible for only $22 \%$ of rapes, whereas husbands and boyfriends were responsible for $19 \%$ and other relatives accounted for $38 \%$. Suggesting that intra-familial sexual abuse is more common than extra-familial sexual abuse. On the opposing end, a study from India revealed that friends/co-students were responsible for $53 \%$ of the sexual abuse, strangers $27 \%$, and relatives/parent responsible for $8 \%$ of sexual assaults within a sample of school going children [2]. Nevertheless, it seems that sexual abuse is highly prevalent in familial settings. Considering the contradictions in the research, a study was conducted to examine the prevalence of intra/extra-familial sexual abuse and the impact it has on a sample of black Zulu speaking children. This was identified as an important study given the dearth of research involving this population.

From as early as 1986 it was believed that the impact of sexual abuse on the child is highly detrimental if the perpetrator is the father or some close relative [3]. Since then more studies have provided evidence that sexual abuse perpetrated by a family member, compared to a stranger, leads to long-term negative consequences and a high level of distress $[4,5,6,7]$. One possible explanation is that sexual abuse perpetrated by a relative often implies that the child will be frequently abused and the contact could potentially last longer, which may result in more severe psychopathology. Few studies have reported that victims abused by an extra-familial perpetrator, compared to those abused by family members, have higher level of symptomatology [8,7). A number of variables are identified as crucial in determining the impact of intra-familial sexual abuse on children. For example a cohesive, supportive family environment is identified as having a potentially buffering effect against the negative impact of sexual abuse [9].

Regarding the type of impact Linda and colleagues [10] 
found that most of the abuse victims develop symptoms, which overlap between complex Post traumatic stress disorder (PTSD) and borderline personality disorder. Explaining complex PTSD Van der Kolk and colleagues [11] suggested that it is a diagnosis, consisting of an admixture of axis I (state) and axis II (trait) symptoms and embodies a constellation of symptoms resulting from early exposure to adverse events.

In examining how the processing of trauma related information occurs following childhood sexual abuse the information processing model was examined. Based on the information processing model, when a traumatic event such as sexual abuse occurs there is: 1) a stimulus/input at the sensory level, followed by; 2) an activation of verbal, behavioural and physiological receptor neurons at the perceptual level; 3) meaning is attached at the cognitive level and; 4) a response is issued at the interpersonal level. Entry of information related to the abusive incident into the brain proceeds from sensory stimulation through to the perceptual level, cognitive level and finally to the interpersonal level. Normal information processing is affected because trauma provides highly salient information which does not fit into pre-existing schema, and successful processing is impaired. Orientation is sometimes affected; patients report forgetfulness, especially concerning the specific details of the event; concentration is affected; impulse control is affected; speech rate and flow is affected; mood and affect changes; thoughts and perception may also be affected.

The information processing theory discussed in the previous paragraph provides an explanation of the pathophysiology of trauma that served as background for the use of neuropsychological screening tests in the study. Whereas the attachment model referred to briefly below provides a more psychological view of trauma. While the information processing theory is broad and flexible in application, it does not adequately provide an explanation for the changes in brain matter which often occur following trauma. For the explanation of changes in the brain matter as a result of exposure to traumatic experiences the reader is referred to the neurobiological model.

Drawing from the attachment theory it was hypothesized that sexual abuse from within the family is expected to be particularly more traumatic for the participants and be associated with severe symptoms of depression, PTSD and cognitive deficits. The attachment theory provides an understanding of the atypical behaviour of individuals exposed to traumatic experiences earlier in life. For the young child whose experiences with attachment figures have been traumatic disorganized attachment occurs resulting in problems with affect regulation, cognitive impairments and a high risk for psychopathology [12].

\section{Methodology}

Results reported in the paper are from 72 children drawn from a clinical population of sexual abuse survivors.
Permission to conduct the research was obtained from the institution involved as well as ethics approval from the collaborating university. Informed consent was obtained from parents. To avoid dual roles, participants who showed significant emotional distress during data collection were contained briefly by the researcher and managed further by other psychologists in the department. Within the descriptive- explorative design, information was collected with the use of the clinical interview, standardized questionnaires and standardized neuropsychological screening tests.

\subsection{Methods of Measurement}

Data collection was divided into four different sessions. The duration of the sessions varied depending on each participant's willingness to talk and motivation to complete the tasks involved. The first session consisted of the provision of information regarding the study, obtaining of informed consent, and conducting the clinical interview. The second session involved the administration of the intellectual assessment tools such as The Ravens Coloured progressive matrices/Standard progressive matrices (CPM/SPM), Children's Depression Inventory (CDI), and The Children's Impact of Events Scale-Revised (CITES-R). The last two sessions involved the administration of assessments of attention, memory and learning. The following battery of tests was used: The Auditory Verbal Learning Test (AVLT); digits forward and backward (DGT-FWD \& DGT-BWD); and The Symbol Digits Modalities Test (SDMT).

\subsection{Analysis}

Psychometric tests were scored individually before any statistical analysis could be done. It is acknowledged that in childhood sexual abuse numerous variables can be included in the analysis of outcome studies. These may include but are not limited to: duration, nature and frequency of the abuse, the child's personality, the relationship between the child and perpetrator, age, and socioeconomic status. However, because of the number of tests involved in data collection and the scores obtained from the sample the statistical analysis was focused on a limited number of variables. The predictor variables selected for analysis were split into two main categories consisting of intra-familial abuser (PV1) and extra-familial abuser (PV2). Participants abused by individuals identified as family members were placed in PV1 and participants abused by individuals hiding their faces at the time of the attack or unknown were placed in PV2. Findings were first scored individually using scoring guidelines set out by test developers and test publishers. Descriptive statistics assisted in summarizing the information obtained to establish an overall impression of the findings. A chi-square analysis was used to establish the presence of relationships between predictor variables and outcome variables. Where groups less than five in each cell were encountered, Fisher's Exact Test results are reported. 


\section{Results}

\subsection{Demographic Variables}

Age of onset of sexual abuse for 42(58\%) participants was before the age of 12 years, and age of onset for $30(42 \%)$ participants was between the age of 13 and 15 years. Twelve children (17\%) did not indicate their level of education, $35(48 \%)$ had an education that was between the first grade and the third grade, and $25(35 \%)$ children had an education that was between grades 4-8. Most of the children 64(89\%) did not receive counselling following the abuse incident and only $8(11 \%)$ received counselling following the sexual abuse incident.

Results showed that 39(54\%) children were abused by a family member and 33(46\%) experienced extra-familial attacks. The frequency distribution indicated that 20(27\%) children were abused by a step-father, $5(7 \%)$ were abused by a biological father, 10(14\%) were abused by an uncle, $4(6 \%)$ were abused by a brother and $33(46 \%)$ were abused by a brother's friend or sister's friend or mom's boyfriend.

\subsection{Depression}

The range of scores obtained from the CDI used to identify symptoms of depression was from 0 to 43 with a mean depression score of $20.9, \mathrm{SD}=16$ which suggests the presence of moderate depression based on Kovacs' scoring guidelines [13]. Cut-off scores were from 0-5 for minimal depression, 6-19 mild depression, 20-28 moderate depression and 29-46 severe depression. The histogram revealed that $48(67 \%)$ children presented with minimal depression, $6(8 \%)$ presented with mild depression, another $6(8 \%)$ with moderate depression and $12(17 \%)$ obtained high scores on the CDI suggesting severe depression. Regarding the subscales for the CDI children reported more symptoms on the negative mood subscale, followed by anhedonia, negative self-esteem, interpersonal problems, and ineffectiveness. Due to the small number of participants in the $2 \times 2$ cells when conducting the chi square analysis, the Fisher's Exact Test yielded more reliable findings. A non-significant $(p=.223,2$-sided Fisher/s Exact Test) relationship was found between predictor variables and depression.

\subsection{PTSD}

The mean total stress score for on the CITES-R was 28.7, $\mathrm{SD}=18.5$ which falls in the range of moderate PTSD. Scores obtained by children were subdivided into four levels. Twenty-one percent of the participants had subclinical levels of PTSD, most 35(49\%) had mild PTSD, 14(19\%) had moderate PTSD and $8(11 \%)$ had severe PTSD. A factorial analysis conducted yielded 11 subscales. When looking at the 11 subscales of the CITES-R, children scored higher on the avoidance subscale $(\mathrm{M}=5.08, \mathrm{SD}=5.57)$ than on any of the other subscales. The lowest score obtained by children on the subscales of the CITES-R was on the social support subscale $($ mean $=1.4, \mathrm{SD}=1.29)$. A significant relationship between

Predictor variables and PTSD was found from a Chi-square analysis $(X 2=5.208, d f=1, p=.022)$ with more children abused by an extra-familial individual presenting with PTSD compared to participants exposed to intra-familial abuse.

\subsection{Cognitive Ability}

Due to the low number of participants in the extreme lower and upper age ranges, participants' scores were collapsed into the mean age of 11.5 which was then used for further analysis. The distribution of raw scores ranged from a minimum score of 8 to a maximum score of 33 with a mean average CPM score of 26 correct items, which placed the score at the 25th percentile yielding an IQ equivalent of 90 on the US norms [14]. Looking at all the participants in the study 39(54\%) children obtained scores below the 50th percentile and $33(46 \%)$ scored above the $50^{\text {th }}$ percentile.

In assessing the relationship between predictor variables and CPM/SPM scores, 25(37\%) participants exposed to intra-familial sexual abuse obtained scores below the 50th percentile and $37(63 \%)$ children who experienced extra-familial sexual abuse obtained scores below the 50th percentile. However, the Pearson Chi-square analysis indicated that there is a non-significant relationship between the scores obtained by the participants on the CPM/SPM and the predictor variables $(X 2=2.824, d f=1, p=.09)$.

\subsection{Attention, Arousal and Mental Tracking Ability}

While the DGT-FWD and DGT-BWD tasks are related, the combination of these tasks into a single score would have obscured important information therefore the results are presented separately. Overall the children obtained a mean score of 4 with a standard deviation of 1.3 on digits Backward with most of them $48(66.7 \%)$ scoring below the mean. On digits forward the mean for the whole group was 4.8 with a standard deviation of 1.70 indicating a borderline performance. On the digits forward test, more participants, 24(73\%) who experienced extra-familial abuse obtained scores below the mean compared to $9(27 \%)$ children in the same category who obtained normal scores. A Chi-square analysis suggested that there is a significant relationship between predictor variables and digits forward $(X 2=15.228$, $d f=1, p=.000)$. Results also pointed towards a significant relationship between predictor variables and digits backward $(X 2=3.915, d f=1, p=.04)$. In copying the SDMT within 120 seconds the average time taken by the participants was 34.4 seconds $(\mathrm{M}=34.4, \mathrm{SD}=12.70)$ which is within the normal range for children.

\subsection{Memory and Learning Ability}

The mean score for the delayed reproduction of the 
complex figure was $17.4, \mathrm{SD}=12.7$ which is below the mean of 22.59, $\mathrm{SD}=6.65$ for normal children. On the AVLT mean scores obtained by the participants on Trial 1, Trial 5 and Trial 7 were (Trial $1[\mathrm{M}=6.80, \mathrm{SD}=1.71]$, Trial $5[\mathrm{M}=$ $12.38, \mathrm{SD}=1.81]$, Trial $7[\mathrm{M}=11.31, \mathrm{SD}=2.03])$ which are lower than mean scores for the normal population of children. A significant relationship was found between predictor variables and the participants' performance on the trial 7 ( $X 2$ $=4.909, d f=1, p=.02$ ). Out of the 39 children abused by a family member $30(77 \%)$ obtained scores within the normal range on the delayed recall trial and $9(23 \%)$ children in the same category obtained scores below average. Similarly, more $29(88 \%)$ of the 33 children abused by an unknown perpetrator obtained low scores compared to $4(12 \%)$ in the same category who obtained normal scores. Overall, significantly fewer words were recalled by the children in the delayed trial compared to the norms of the normal population.

\section{Discussion}

Although the CDI is a good indicator of self-reported depressive symptoms in children the current study demonstrated that it does not have adequate sensitivity. In this study a clinical sample was used and higher rates of depression were expected therefore different cut off points were identified to increase sensitivity and specificity of the scale thus eliminate false positives. In spite of that, most children reported subclinical symptoms of depression and obtained scores between 0 and 18 which is below a score of 19 identified by Kovacs as indicative of a diagnosis of depression. Findings in the current study concur with Kendall-Tackett and colleagues [15] who found that while many sexually abused children present with symptom specific distress there is still a substantial group who remain asymptomatic. Low levels of depression in children are consistent with the information processing theory's proposition that in childhood sexual abuse, a disruption in the processing of the sexual abuse experience occurs. Disruptions at the perceptual level of information processing result in a lack of visual memory, and the children fail to attach meaning to the sexual abuse experience at the cognitive level and the response shown lacks the affective component.

While the CITES-R scores also were not suggestive of serious psychopathology in children, the literature indicates that there are a variety of factors involved in the response to sexual abuse and being symptom free does not necessarily indicate that the child was not affected by the abuse. This finding is consistent with the propositions of the Child Accommodation Syndrome which suggests that children may dissociate and disengage from the traumatic memories as a way of protecting themselves from the impact of the trauma. In so doing they remain without any symptoms of PTSD or depression. In addition it has previously been observed that in children PTSD may present as other disorders such as Attention Deficit Hyperactivity Disorder
(ADHD), Conduct Disorder, Anxiety Disorders and Mood Disorders [16].

Collectively, low scores were obtained by participants on the tests of attention and mental tracking. Findings also support propositions of the information processing model in that when a child struggles with input from sexual abuse, she/he cannot deal successfully with anything beyond the input level. This block in information processing could have affected performance on the CPM and other screening measures used in the study $[17,18]$.

In summary, findings showed that more participants exposed to extra-familial sexual abuse had more symptoms of depression, PTSD and cognitive deficits. This finding is in line with the Betrayal trauma theory proposed by Freyd in 1994. The theory suggested that children abused by a family member choose to promote a need for survival and dissociate themselves from the traumatic memory. In so doing they retain their attachment to the abuser so as to receive the care that they need. A need for survival affects the child's awareness and memory of the abuse which consequently affects symptoms reported by the child [19].

\section{Limitations and Strengths of the Study}

Results need to be interpreted with caution as all psychometric tests used were not standardized for the Black South African population. For example most of the children in the current study obtained IQ scores below an average IQ of 100 on the CPM. However, this finding needs to be explored further, given that it was based on US norms. When using Lindstrom [20] South African norms, the same mean score of 26 correct items for the $\mathrm{CH}$ group falls at the 50th percentile with an IQ equivalent of 100 . When a further adjustment is made with the South African norms for Xhosa speaking children, the score of 26 falls at the 75 th percentile with a mean IQ of 110 [21]. While the use of South African norms would have been better suited for the population studied, the variation in the norms is an issue of great concern and a need for valid and reliable South African norms for the rainbow nation is emphasized. Sample size is also a factor that could have resulted in low scores and and failure to find significant relationships in some of the variables which represents a Type II error. However, the selection of participants from a population of childhood sexual abuse survivors can be considered a positive factor. This ensured external validity (the degree to which correct and reliable inferences can be drawn from this study when generalizing to other sexual abuse survivors). Because of the nature of the descriptive exploratory approach, another limitation of this study is that causation cannot be stipulated for sure but can be inferred.

\section{Conclusions}


Reflecting on the findings, this study contributes to the literature in the following manner:

The observation that children rely on dissociation and simply disengage from traumatic stimuli has been endorsed. Another observation is that when children seem to be doing well from assessments using rating scales and do not present with psychopathological symptoms following traumatic events it does not necessarily mean that the trauma had no impact on them, instead there is a need for use of alternative assessment measures such as neuropsychological tests. It is also concerning that most of the children in the study did not receive counselling following the attack. This finding highlights the need for a review of intervention procedures available in the country for survivors of sexual abuse.

Based on the above conclusions the following recommendations are made: The study calls for an innovative approach to the assessment of children following traumatic events. The study calls upon the reader to look beyond the absence or presence of depression and PTSD found in the sample, but to look at the bigger picture - the violation of human rights and lack of adequate resources for intervention.

\section{REFRENCES}

[1] D. G. Kilpatrick, C. N. Edmunds., A, K. Seymour. Rape in America: A Report to the Nation, Artington, VA, National Victim Centre, 1992.

[2] M. M. Firdosi, M. A. Margoob. Varying clinical presentation in victims of sexual traumatization, JK-Practitioner, Vol.13, No.1, 73-78, 2006

[3] A. D. Browene, D. Finkelhor. Impact of child sexual abuse: Review of the research, Psychological Bulletin, Vol.99, 66-77, 1986.

[4] G. E. Wyatt, M. Newcomb. Internal and external mediators of women's sexual abuse in childhood, Journal of Consulting and Clinical Psychology, Vol.58,758-767, 1991.

[5] J. Faust, M. K. Runyon, M. C. Kenny. Family variables associated with the onset and impact of interfamilial childhood sexual abuse, Clinical Psychology Review, Vol.15, 443-456, 1995.

[6] D. G. Fisher, W. L. McDonald. Characteristics of intrafamilial and extrafamilial child abuse, Child Abuse and Neglect, Vol.22, 915-929,1998.

[7] B. A. Lucenko, S. N. Gold, M. A. Cott. Relationship to perpetrator and post traumatic symptomatology among sexual abuse survivors. Journal of Family violence, Vol.15, 169-179,2000.

[8] C. A. Grosz, R. S. Kemp, M. Kelly. Extrafamilial sexual abuse: Treatment of child sexual abuse victims and their families, Child Abuse and Neglect, Vol.24, 9-23, 1995.

[9] K. C. Ray, J. L. Jackson. Family environment and childhood victimization: A test of buffering hypothesis, Journal of Interpersonal violence, Vol.12, 3-17, 1997.

[10] L. M. McLean, R. Gallop. Implication of childhood sexual abuse for adult Borderline Personality disorder and Complex PTSD, American Journal of Psychiatry, Vol.160, 369-371, Feb 2003.

[11] B. A. Van der Kolk, D. Pelcovitz, S. Roth, F. S. Mandel, A. McFarlane, J. L. Herman. Dissociation, somatization, and affect dysregulation: the complexity of adaptation to trauma, American Journal of Psychiatry, Vol.153, 83-93, 1996.

[12] P. M. Crittenden, M. D. S. Ainsworth. Child maltreatment and attachment theory, 432 - 463, 2010. In Child maltreatment: Theory and research on the causes and consequences of child abuse and neglect. D. Cicchetti \& V. Carlson (Eds). Chapter DOI available online from: http://dx.doi.org/10.1017/CBO9780511665707.015

[13] M. Kovacs'. Children's depression inventory. New York: Multi Health

[14] Systems Inc., 2003.

[15] J.C. Raven,J. C. Raven, J. H. Court. Manual for Ravens progressive Matrices and Vocabulary Scales- Section 2: Coloured Progressive Matrices. Oxford: Oxford Psychologists Press, 2000.

[16] K. A. Kendall-Tacket, L. M. Williams, D. Finkelhor. Impact of sexual abuse on children: A review and synthesis of recent empirical studies, Psychological Bulletin, Vol.113, 164-180, 1993.

[17] L. C. Terr. Childhood traumas: An outline and overview, American Journal of Psychiatry, Vol.148, 10-20, 1991.

[18] E. Vakil, H. Blachstein, M. Sheinman, Y. Greenstein. Developmental changes in attention tests norms: Implications for the structure of attention, Child Neuropsychology, 15, 21-39, 2009. Available online from: http://www.psychpress.com/childneuropsych

[19] P. Ogden, K. Minton, C. Pain. Hierarchical information processing: cognitive, emotional and sensorimotor dimensions, 2006. In P. Ogden (Ed). Trauma and the bossy: a sensorimotor approach to psychotherapy. New York: W. W. Norton.

[20] J. J. Freyd. Betrayal trauma: Traumatic amnesia as an adaptive response to childhood abuse, Ethics and Behavior, Vol.4, No.4, 307-329, 1994.

[21] A. Lindstrom, J. Raven, J. Raven. The Coloured Progressive Matrices in South Africa. In J. Raven and J. Raven (Eds). Uses and abuses ofintelligence: Studies advancing Searman and Raven's quest for non-arbitrary Metrics. New York: Royal Fireworks Press. Retrieved from: http://www.iapsych.com 2008.

[22] J. Knoetze, N. Bass, G. Steele. The Raven's Progressive Matrices: Pilot norms for isiXhosa-speaking primary school learners in peri-urban Eastern Cape. South African Journal of Psychology, 35(2), 175-194, 2005. 